\title{
Reactive properties of bioengineered and natural iron oxides: a multi-scale approach from first principles
}

\author{
I. KuRganskaya ${ }^{1 *}$, M. SChÜLER ${ }^{2}$, C. SANTIAGO ${ }^{1}, \mathrm{~T}$. \\ WEHLING $^{2}$ AND A. LUTTGE ${ }^{1}$ \\ ${ }^{1}$ MAPEX \& FB5, Universität Bremen, 28359 Bremen, \\ Germany (*inna.kurganskaya@uni-bremen.de) \\ 2 Institute for Theoretical Physics and Bremen Center for \\ Computational Materials Science, Universität Bremen, \\ 28359 Bremen, Germany
}

Iron oxides, hematite and magnetite, are ubiquitous minerals in Earth's surface and subsurface environments. They can be produced via abiotic or biogenic pathways, e.g., by magnetotactic bacteria [1], bacteria producing hierarchical nano-composite structures [2]. Biogenic nanoparticles have a

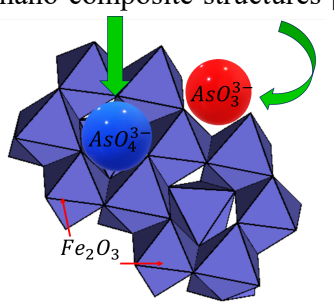
variety of important environmental applications, such as the cleaning of As-polluted drinking water [3]. Dissolution mechanisms of iron oxides at nano and larger scales are obscure due to coupled dissolution and $\mathrm{Fe}^{3+} /$ $\mathrm{Fe}^{2+}$ electron transfer reactions [4]. We employ Kinetic Monte Carlo (KMC) simulations to decypher nano-scale dissolution and As adsorption mechanisms for natural and bioengineered hematite nano-particles, as well as surfaces of micron-cm sized hematite particles. The validation of the model is based on complementary Atomic Force Microscopy and Vertical Scanning Interferometry studies of dissolving hematite surfaces and their characterization for potential adsorption sites.

As we demonstrated earlier [5], parameterization of a KMC model is a critical step affecting its predictive capacity. The use of the molecular-scale rates derived from $a b$ initio calculations is the preferred method of parameterization. Transition metal oxides attracts a great attention from physisists due to strong electron-electron correlation effects [6]. We adopt the corresponding novel electronic structure methods [6] to study bond-breaking mechanisms on hematite and magnetite surfaces and calculate molecular reaction rates.

[1] Yan et al. (2017) Microbiol. Res. 203, 19-28. [2] Kim et al. (2019) ACS Appl. Mater. Interfaces 11 (7), 6948-6957. [3] Yavuz et al. (2010) Environ Geochem Health 32, 327-334. [4] McBriarty et al. (2019) J. Phys. Chem. C 123, 8077-8085. [5] Kurganskaya \& Luttge (2013) J. Phys. Chem. C 117, 24894-24906. [6] Schüler et al. (2018) J. Phys.: Condens. Matter 30 (47), 475901. 